\title{
CONGRUENCES INVOLVING GENERALIZED FROBENIUS PARTITIONS
}

\section{JAMES SELLERS}

\author{
Department of Science and Mathematics \\ Cedarville College \\ Cedarville, OH 45314
}

(Received June 19, 1992 and in revised form July 10, 1992)

\begin{abstract}
The goal of this paper is to discuss congruences involving the function $\overline{c \phi_{m}}(n)$, which denotes the number of generalized Frobenius partitions of $n$ with $m$ colors whose order is $m$ under cyclic permutation of the $m$ colors.
\end{abstract}

KEYWORDS AND PHRASES. Congruence, partitions. 1991 AMS SUBJECT CLASSIFICATION CODE. 05A17, $11 \mathrm{P} 83$.

\section{INTRODUCTION.}

In 1984, George Andrews [1] introduced the idea of generalized Frobenius partitions, or Fpartitions, and discussed many of the properties associated with them. In particular, he studied the function $c \phi_{m}(n)$, the number of F-partitions of $n$ with $m$ colors. One of the results that Andrews obtained was the following: If $m$ is prime then

$$
c \phi_{m}(n) \equiv 0 \quad\left(\bmod m^{2}\right)
$$

for all $n \geq 1$ not divisible by $m$.

More recently, Louis Kolitsch [2,3] has considered the function $\overline{c \phi_{m}}(n)$, which denotes the number of F-partitions of $n$ with $m$ colors whose order is $m$ under cyclic permutation of the $m$ colors. Kolitsch has proven that, for $m \geq 2$ and for all $n \geq 1$,

$$
\overline{c \phi_{m}}(n) \equiv 0 \quad\left(\bmod m^{2}\right)
$$

\section{MAIN RESULT.}

We now want to prove the following congruence related to (1.2).

THEOREM 1: For $m=5,7$, and 11, and for all $n \geq 1$,

$$
\overline{c \phi_{m}}(m n) \equiv 0 \quad\left(\bmod m^{3}\right)
$$

Proof: In [3], Kolitsch proved that, for all $n \geq 1$,

$$
\begin{aligned}
& \overline{c \phi_{5}}(n)=5 p(5 n-1), \\
& \overline{c \phi_{7}}(n)=7 p(7 n-2), \text { and } \\
& \overline{c \phi_{11}}(n)=11 p(11 n-5)
\end{aligned}
$$


where $p(n)$ is the ordinary partition function. Now we note that

$$
\begin{aligned}
\overline{c \phi_{5}}(5 n) & =5 p(25 n-1), \\
\overline{c \phi_{7}}(7 n) & =7 p(49 n-2), \text { and } \\
\overline{c \phi_{11}}(11 n) & =11 p(121 n-5) .
\end{aligned}
$$

Moreover, several authors have shown that

$$
\begin{aligned}
p(25 n-1) & \equiv 0\left(\bmod 5^{2}\right), \\
p(49 n-2) & \equiv 0\left(\bmod 7^{2}\right), \text { and } \\
p(121 n-5) & \equiv 0\left(\bmod 11^{2}\right) .
\end{aligned}
$$

(See Andrews [4] for an excellent discussion of these congruences first noticed by Srinivasa Ramanujan.) Hence, we see that

$$
\begin{aligned}
\overline{c \phi_{5}}(5 n) & \equiv 0\left(\bmod 5^{3}\right), \\
\overline{c \phi_{7}}(7 n) & \equiv 0\left(\bmod 7^{3}\right), \text { and } \\
\overline{c \phi_{11}}(11 n) & \equiv 0\left(\bmod 11^{3}\right),
\end{aligned}
$$

This is the desired result.

\section{FINAL REMARKS.}

Now it would appear that congruences like (2.1) above hold for other values of $m$ as well. This author has considered congruences of the form above for $m=2$ and 3 . Values involving $\overline{c \phi_{2}}(2 n)$ and $\overline{c \phi_{3}}(3 n)$ have been found for several values of $n$, which were easily computed using the generating functions for $c \phi_{2}(n)$ and $c \phi_{3}(n)$ developed in [1] and the fact that

$$
\overline{c \phi_{m}}(m n)=c \phi_{m}(m n)-p(n)
$$

for prime $m$. Given these, it appears that the following congruences hold:

Conjecture: For all $n \geq 1$,

$$
\begin{aligned}
& \overline{c \phi_{2}}(2 n) \equiv 0\left(\bmod 2^{3}\right) \text { and } \\
& \overline{c \phi_{3}}(3 n) \equiv 0\left(\bmod 3^{3}\right) .
\end{aligned}
$$

It may be possible that such a congruence holds for each prime $m$, although this author has not pursued this. 
VALUES OF $\overline{c \phi_{2}}(2 n)$ AND $\overline{c \phi_{3}}(3 n)$

$\begin{array}{rrr}n & \overline{c \phi_{2}}(2 n) & \overline{c \phi_{3}}(3 n) \\ 1 & & 81 \\ 2 & 8 & 1053 \\ 3 & 40 & 8424 \\ 4 & 144 & 50625 \\ 5 & 440 & 252720 \\ 6 & 1208 & 1099332 \\ 7 & 3048 & 4301667 \\ 8 & 7224 & 15451722 \\ 9 & 16264 & 51712830 \\ 10 & 35080 & 162997272 \\ 11 & 72968 & 487927557 \\ 12 & 147088 & 1396216926 \\ 13 & 288424 & 3839379507 \\ 14 & 551936 & 26189278765 \\ 15 & 1033360 & 26191056294 \\ 16 & 1896912 & 6402440254 \\ 17 & 3420296 & 159066295911 \\ 18 & 6066968 & 377624881413 \\ 19 & 10601000 & 876738665745 \\ 20 & 18268120 & 1994026912767 \\ 21 & 31078000 & 4449189414618 \\ 22 & 52241184 & 9751794680439 \\ 23 & 86839912 & 21020605245324 \\ 24 & 142850088 & 44608075732350 \\ 25 & 232687400 & 93281355133110 \\ 26 & 375531240 & 192378123793026 \\ 27 & 600794432 & 391587178790619 \\ 28 & 953273544 & 787255913193255 \\ 29 & 1500749624 & 1564208883888750 \\ 30 & 3645143040 & 3073396018972779 \\ 31 & 5608145688 & 597459684687374 \\ 32 & 8587893472 & 11497819468200462 \\ 33 & 13070249344 & 21913027419434670 \\ 34 & 19775421160 & 41377597875587103 \\ 35 & 29752192096 & 77441754423150981 \\ 36 & 44520802024 & 143711420261068 \\ 37 & 66275131408 & 264522134520406248 \\ 38 & 98167705768 & 483087841030377438 \\ 39 & 144709970880 & 875615409510183201 \\ 40 & 212332459688 & 1575598824183500991\end{array}$

\section{REFERENCES}

1. ANDREWS, G. E., Generalized Frobenius Partitions, Memoirs of the American Mathematical Society, Volume 301, Providence, RI, May 1984.

2. KOLITSCH, L., An Extension of a Congruence by Andrews for Generalized Frobenius Partitions, J. Combin. Theory Ser. A $\underline{45}$ (1987), 31-39.

3. KOLITSCH, L., M-Order Generalized Frobenius Partitions With M Colors, J. Number Theory $\underline{39}$ (1991), 279-284.

4. ANDREWS, G. E., The Theory of Partitions, Encyclopedia of Mathematics and Its Applications, Volume 2, Addison-Wesley, Reading, MA, 1976. 


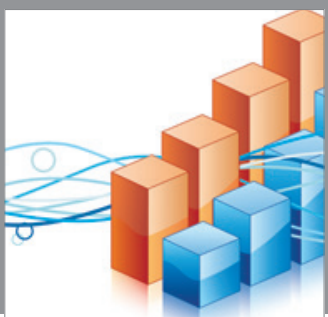

Advances in

Operations Research

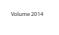

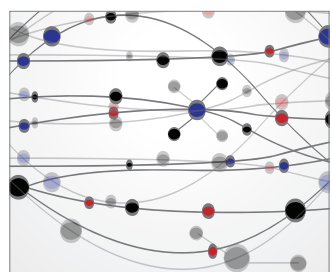

\section{The Scientific} World Journal
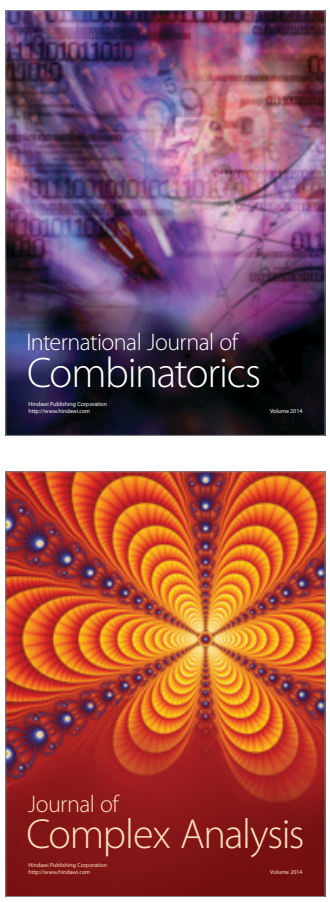

International Journal of

Mathematics and

Mathematical

Sciences
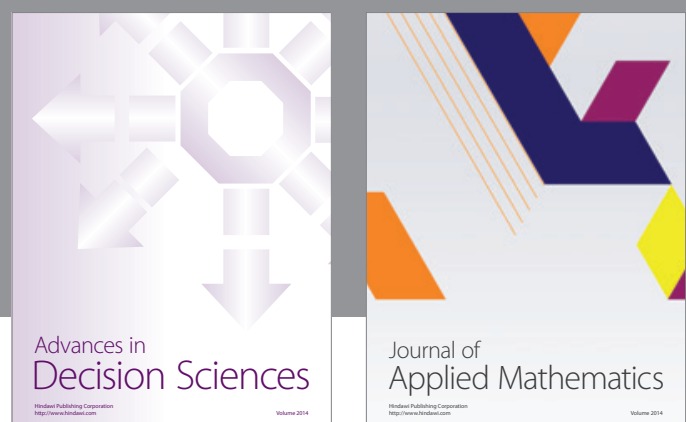

Journal of

Applied Mathematics
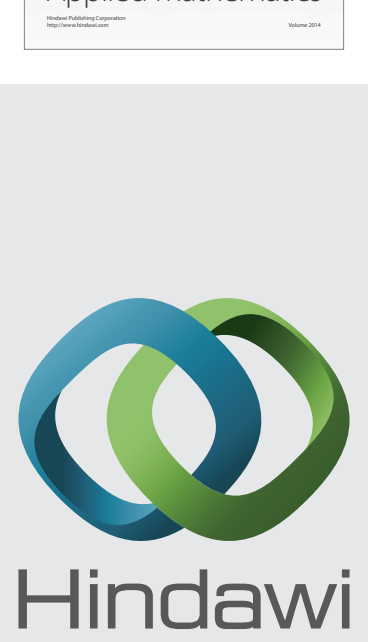

Submit your manuscripts at http://www.hindawi.com
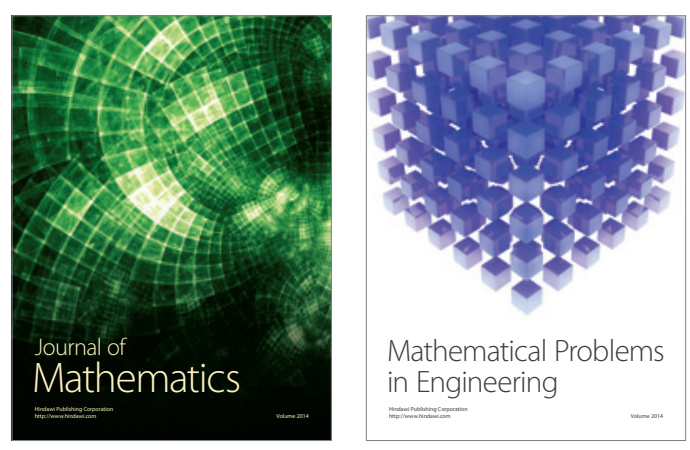

Mathematical Problems in Engineering
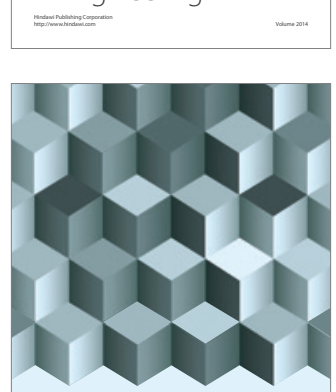

Journal of

Function Spaces
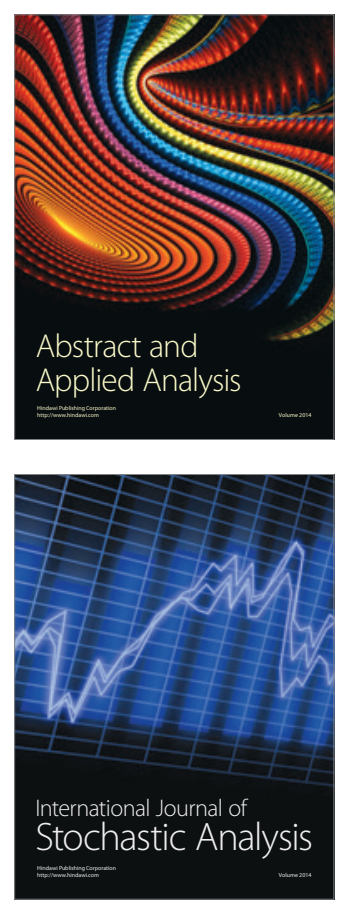

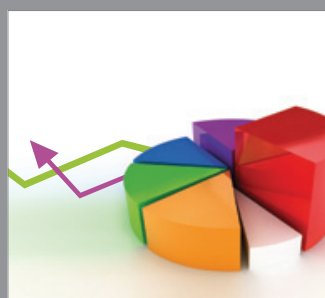

ournal of

Probability and Statistics

Promensencen
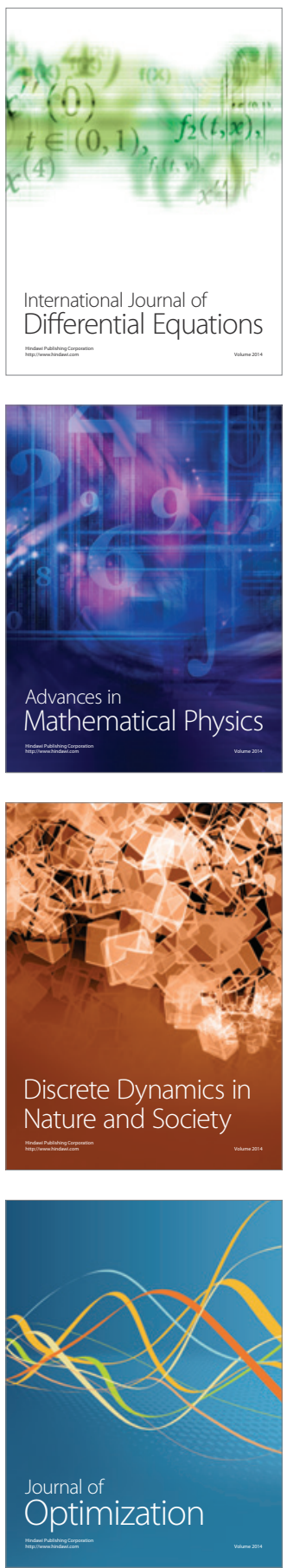